\title{
Depressionsbehandlung in der Hausarztpraxis
}

\author{
Therapy of Depression in Primary Care
}

\section{Bibliografie}

Dol http://dx.doi.org/

10.1055/s-0030-1248585

Psychiat Prax 2010; 37:

366-368

(c) Georg Thieme Verlag KG

Stuttgart · New York .

ISSN 0303-4259

\section{Korrespondenzadressen}

Prof. Dr. med., Dipl..Päd. Jochen Gensichen, MPH

Institut für Allgemeinmedizin Universitätsklinikum Jena Bachstraße 18

07743 Jena

jochen.gensichen@med.unijena.de

\section{Dr. med. Sigrid}

Planz-Kuhlendahl

Fachärztin für Neurologie

Psychiatrie und Psychotherapie Aliceplatz 7

63065 Offenbach

planz-kuhlendahl@t-online.de

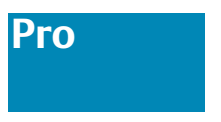
Hausärzte stellen im Gesundheitswesen in der Regel die primäre Anlaufstelle für Patienten dar. Auch bei der Behandlung von Depressionen spielt das hausärztliche Setting eine große Rolle. So werden etwa ein Drittel bis die Hälfte aller depressiven Erwachsenen und sogar zwei Drittel der depressiv älteren Patienten hausärztlich behandelt [1].

\section{Diagnosestellung}

Die Erstdiagnose einer Depression stellt häufig eine Herausforderung für den behandelnden Arzt dar, da etwa zwei Drittel der Patienten sich zunächst mit unspezifischen Symptomen vorstellen. Neben den „klassischen“ Haupt- und Nebendiagnosekriterien nach ICD-10 und DSM-IV einer Depression gibt es eine Reihe weiterer Signalsituationen (engl. „red flags“) in der hausärztlichen Praxis, in welchen der Hausarzt eine strukturierte Depressionsdiagnostik durchführen sollte, so z.B. bei häufigem Sprechstundenbesuch mit wechselnden Beschwerden, bei Beschwerden ohne körperliche Ursache, bei chronischen Schmerzen, bei Nervosität oder Schlaflosigkeit, bei neu aufgetretenem Verschreibungswunsch von Schlaf- und Beruhigungsmitteln etc. [2,3].

Der Hausarzt hat hier gegenüber dem Psychiater den Vorteil, dass die Patienten in der Regel bereits längerfristig bei ihm in Behandlung sind und eine vertrauensvolle Beziehung zu ihm aufgebaut haben. Viele sieht er regelmäßig, beispielsweise im Rahmen von Früherkennungsuntersuchungen oder bei der Verschreibung von neuen Rezepten. Diese Umstände sowie die in der Regel gute Erreichbarkeit durch räumliche Nähe können dem Patienten einen Besuch beim Hausarzt erleichtern. Durch die Rolle als „Familienarzt“ kennt der Hausarzt zudem das familiäre Umfeld und erhält wichtige Informationen durch Angehörige („Mein Mann hat sich so verändert, Herr Doktor“) und weiß um belastende Ereignisse (Verlust des Ar- beitsplatzes, Tod eines nahen Angehörigen etc.). Im hausärztlichen Praxisalltag bieten sich somit eine Reihe von Situationen, um auf solche „red flags“ aufmerksam zu werden und damit einer nicht direkt präsentierten Depression zu erkennen.

Handelt es sich dagegen um eine rezidivierende Episode einer Depression oder kennt der Patient seine Krankheit und wünscht eine Betreuung durch (s)einen Psychiater, muss ihm dies selbstverständlich ermöglicht werden.

Wiederholt werden Hausärzte wegen unzureichender oder verspäteter Diagnosestellung von Depressionen kritisiert - so zeigen ältere Studien, dass bis zu 50\% der Patienten vom Hausarzt nicht adäquat erkannt werden. Diese Untersuchungen beziehen sich aber zumeist nur auf eine Stichtagsuntersuchung, was nicht der Realität in der Hausarztpraxis entspricht. Tyrer etwa kann in seiner Arbeit von 2009 zeigen, dass die hausärztliche Einschätzung des longitudinalen Gesamtbildes reinen Screeningfragen überlegen ist [4].

\section{Therapie und Betreuung depressiver Patienten}

Nach Diskussionen um die Wirksamkeit und Indikation von Antidepressiva und der damit einhergehenden Verunsicherung bezüglich der optimalen Pharmakotherapie für depressive Patienten konnten Ende 2009 mit der S3- bzw. Nationalen Versorgungsleitlinie „Unipolare Depression“ evidenzbasierte Empfehlungen für Deutschland gegeben werden, die zuvor von allen relevanten Fachgesellschaften konsentiert wurden $[5,6]$.

Die Empfehlungen dieser Leitlinie sehen bei einer leichten Depression nach Aufklärung und Psychoedukation eine sogenannte „aktiv-abwartende Begleitung“ über 14 Tage vor - ein für den Hausarzt typischerweise vertrautes Vorgehen (engl. „watchful waiting“). Als weitere Therapieoption bei anhaltender oder sich verschlechternder Symptomatik wird dann die Überweisung zum 
Psychotherapeuten empfohlen. Eine antidepressive Pharmakotherapie sollte bei der leichten Depression eher zurückhaltend eingesetzt werden. Bei Präferenz des Patienten, zuvorigem guten Ansprechen auf ein Antidepressivum oder einer Vorgeschichte mit mittelschwerer bis schwerer Depression sollte selbstverständlich eine Pharmakotherapie erwogen werden.

Inwieweit bei fehlendem Erfolg der Pharmakotherapie eine Modifikation (Erhöhung der Dosis, Umstellung des Medikaments etc.) dann noch durch den Hausarzt durchgeführt wird, hängt sicherlich von seinen Erfahrungen und Vorkenntnissen ab. Auch bei fehlender Besserung trotz psychotherapeutischer Behandlung sollte spätestens nach 3 Monaten ein psychiatrischer Kollege hinzugezogen werden. Weitere Indikationen für eine Überweisung zum Facharzt sind eine schwere oder psychotische Depression, komplizierte Verläufe und Suizidalität. Hier ist ggf. auch eine sofortige stationäre Einweisung angezeigt. Auch bei Unsicherheit in der Diagnose oder bei Vorliegen von komorbiden anderen psychischen Störungen sollte der Hausarzt den psychiatrischen Kollegen hinzuziehen.

Dem Hausarzt obliegt eine koordinierende Funktion, was auch bei der Behandlung der Depression von Bedeutung ist. Hier liegt die Bedeutung v.a. in der verbesserten Koordination von schnittstellenübergreifenden Behandlungen (z.B. beim Übergang von stationärer zu ambulanter Behandlung).

\section{Optimierung der hausärztlichen Versorgung depressiver Patienten}

Mittels Case-Management durch Medizinische Fachangestellte in Hausarztpraxen lässt sich die Einhaltung der Therapie (engl. adherence) und der Verlauf der Depression günstig beeinflussen. In der Studie „Primary Care Monitoring for Depressive Patients Trial“ wurden die Symptome durch regelmäßige telefonische Anrufe bei depressiven Patienten durch speziell geschulte medizinische Fachangestellte zusätzlich (!) zur ärztlichen Betreuung signifikant verbessert [7].

\section{Suizidalität in der Hausarztpraxis}

Die Patientengruppe, die am häufigsten von Suizidalität betroffen ist, sind Patienten mit Depressionen - je schwerer die Depression, umso höher das Risiko für Suizidalität. Etwa 75\% der Patienten, die Suizid begehen, haben innerhalb des letzten Jahres und fast die Hälfte innerhalb eines Monats vor ihrem Tod ihren Hausarzt aufgesucht. Daher wird der hausärztlich-ambulanten Versorgung für die Suizidprävention ein besonderes Potenzial zugesprochen [8].

Problematisch bei der Betreuung depressiver Patienten ist nach wie vor die gefürchtete Stigmatisierung durch eine psychische Erkrankung. Auch sind Vorurteile wie „Antidepressiva machen abhängig“ noch weitverbreitet und erschweren eine adäquate Therapie. Durch Projekte wie das „Bündnis gegen Depression“ wird hier erfolgreich Aufklärungsarbeit geleistet und versucht, Vorurteile und Ängste in der Bevölkerung abzubauen [9]. Der Hausarzt stellt dabei eine niedrigschwellige Anlaufstelle dar und erreicht somit auch die Patienten, die bei oft langen Wartezeiten und z.T. schlechter Erreichbarkeit (insbesondere im ländlichen Raum) anderer Versorger, Hilfen brauchen.

Nur durch die enge Kooperation zwischen dem haus- und fachärztlichen sowie den psychotherapeutischen Professionen können depressive Patienten in unserem Gesundheitssystem aber letztendlich optimal versorgt werden.

\section{Kontra}

Die Behandlung psychischer Erkrankungen gewinnt in Deutschland immer mehr an Bedeutung - sowohl im ambulanten als auch im stationären Behandlungsbereich. Inzwischen hat die Häufigkeit psychischer Erkrankungen die durch Herz-Kreislauf-Erkrankungen überflügelt, und die Tendenz ist steigend. Unter den psychischen Erkrankungen stellen die verschiedenen Depressionsformen mit die häufigste Krankheitsgruppe dar; deshalb lautet die Kardinalfrage: Wer soll hier diagnostizieren, wer soll behandeln, wie lange und mit welchem Ziel?

Gegen eine Behandlung von leichten bis mittelschweren depressiven Erkrankungen durch den jeweiligen Hausarzt, der das Vertrauen seines Patienten genießt und dessen soziales Umfeld besser kennt als andere Ärzte, ist überhaupt kein Argument vorzubringen; sie ist notwendig und erhält immer mehr Gewicht in Zeiten, da die Verbreitung der depressiven Erkrankungen laut WHO im Jahre 2020 einen vorläufigen Höhepunkt erreichen soll. Aber der Hausarzt kann in aller Regel nicht der Fachmann sein, da er als Lotse für viele Krankheiten zuständig ist und deren Diagnostik und Therapie allenfalls in die richtigen (Facharzt-)Bahnen lenken kann.

In der Regel kann ein Hausarzt nur leichte depressive Zustände behandeln - und diese sind schwer zu erkennen. Ihre Diagnostik erfordert eine ausführliche Exploration mit vielen behutsam gestellten Fragen, die Geduld und Zeit erfordern und den Hausarzt im Allgemeinen überfordern dürfte. Deshalb wird der engagierte Hausarzt beizeiten einen entsprechend versierten Facharzt zu Rate ziehen, um sich damit den Rücken freizuhalten für andere akute medizinische Probleme.

Der Umgang mit Antidepressiva stellt eine weitere Klippe dar: einziges Antidepressivum ist meist Citalopram, das dann auch noch zur falschen Tageszeit verordnet wird. Über die Interaktion von SSRI, v.a. von Paroxetin, mit internistischen Medikamenten, ist dem Hausarzt meist gar nichts bekannt. Trizyklika scheinen von der Palette der Medikamente fast völlig gestrichen worden zu sein. Ein differenzierter Umgang mit und ein versierter Gebrauch von Antidepressiva ist dem Hausarzt eher fremd - es sei denn, er hat die psychopharmakologische Behandlung zu seinem beruflichen Steckenpferd gemacht.

Die Behandlung von depressiven Patienten kann darüber hinaus eine erhebliche psychische Belastung für den Behandler darstellen, die er oft nur durch den Besuch einer Supervisions- oder Balint-Gruppe schultern kann. Oft wissen Hausärzte gar nicht, dass es solche Gruppen gibt oder wo sie zu finden sind. Und Zeit und Energie eines Hausarztes, der auch noch Hausbesuche und nächtliche Bereitschaftsdienste wahrnehmen muss, ist natugemäß begrenzt.

Wogegen ich mich aber ausdrücklich ausspreche, ist die Diagnostik und eventuelle Therapie durch eine nichtärztliche Mitarbeiterin, ganz gleich ob beim Haus- oder Nervenarzt beziehungsweise beim Psychiater.

So sind mir Vorgehensweisen bekannt, bei denen eine Arzthelferin oder medizinische Fachangestellte des Hausarztes die als depressiv erkannte Patienten in festgelegten Abständen telefonisch kontaktiert und mittels eines vorgegebenen Fragebogens zum jeweiligen Befinden befragen soll; dabei sollte sie u.a. den Patienten (der dafür natürlich zuvor sein Einverständnis schriftlich erklärt hatte) darüber explorieren, ob er Suizidgedanken hege oder gar Suizidimpulse verspüre oder bereits umgesetzt habe. Ab einer bestimmten Punktzahl sollte sie dann den Hausarzt alarmieren, der dann weitere diagnostische und therapeutische Schritte zu veranlassen haben würde. 
Für ein solches Procedere sprechen aus Sicht der Krankenkassen vor allem Kostengründe: Das Vorgehen bei depressiven Patienten mit Telefonanrufen würde den Hausarzt entlasten und die Wartezimmer leeren. Der Hausarzt würde vermutlich besser entlohnt werden in einem solchen Projekt, in dem er depressive Patienten nicht mehr im Rahmen seines schmalen Regelleistungsvolumens in seiner Praxis behandeln müsste. Die Krankenkasse könnte ihren Versicherten das Gefühl vermitteln, sie kümmere sich auch um ihre psychischen Belange und treibe die Intensivierung der Behandlung voran. Ein enormer Imagegewinn wäre die Folge für sie.

Aufseiten des Hausarztes wären aber deutlich mehr versierte, sprachlich und emotional geschulte Fachangestellte notwendig, um überhaupt einen adäquaten Kontakt zu dieser heiklen und oft schwer erkrankten Klientel zu gewährleisten - und diese „Praxisperlen“ sind dünner gesät als Süßwasserperlen in südostasiatischen Gewässern.

Ich fand und finde: ein solches Vorgehen geht zu weit bzw. schießt ins Leere, statt die vielfältig anstehenden brisanten Probleme adäquat zu lösen! Derart heikle, die persönliche Intimsphäre berührenden Themenkomplexe wie eine depressive Verfassung, das Ansprechen auf Medikamente oder gar das Vorliegen von Suizidimpulsen kann man in keiner Weise telefonisch abfragen. Die Erörterung von Suizidalität erfordert einen persönlichen ärztlichen Kontakt des Patienten, und zwar mit dem Arzt seines Vertrauens. Die Schwelle, sich eigenen Suizidideen zu stellen, ist bei den meisten Menschen sehr hoch angesiedelt, und die Beschäftigung damit erfordert größte Sensibilität und ein enormes Fingerspitzengefühl bei dem jeweiligen Gesprächspartner. Das können nichtärztliche Mitarbeiter meist nicht aufbringen, und schon gar nicht telefonisch! Zudem ist die Wahrscheinlichkeit, dass Suizidtendenzen bagatellisiert und dissimuliert werden und sich ihrer Beobachtung und deren Beurteilung entziehen, in einem telefonischen Kontakt höher als in der direkten Exploration, wo zum akustischen das visuelle Moment hinzukommt. Die visuelle Präsenz könnte bestenfalls durch die Installation einer Kamera in der Wohnung des jeweiligen Patienten gesichert werden - und eine solche „Kamerainstallierung“ wollten nicht einmal viele meiner Parkinson-Patienten dulden, als es um ein Projekt zur besseren Steuerung der Parkinson-Medikation ging.

Ein solches Vorgehen ist anders zu bewerten, als der Kontakt zu einer Telefonseelsorge; im letzteren Falle kontaktiert der verzweifelte, suizidale Mensch aus eigenem Antrieb und teilweise mit letzter Kraft die Einrichtung, die dann eine globale Beratung bereitstellen kann. Ein depressiver Patient in der hausärztlichen Praxis könnte sich trotz vorher erteilter Zustimmung durch tele- fonisches und u.U. hartnäckiges „Nachhaken“ erheblich belästigt oder sogar bedrängt fühlen, ohne sich dann aufgrund seiner Erkrankung dagegen zur Wehr setzen zu können - zudem in einem Zustand, in dem sein Selbstwertgefühl am Boden liegt.

Zudem werden Hausarzt und Patient in einer trügerischen Sicherheit gewiegt, dass ihre Krankheit nun „besser im Griff“ liege als bei herkömmlicher Behandlung. Gewogen - und zu leicht befunden, könnte man über ein solches Ansinnen befinden.

Die Behandlung depressiver Patienten und sonstiger psychisch erkrankter Personen gehört in die Hand eines versierten Arztes und sollte nicht den Fingern einer „Schwester Agnes“ überlassen werden.

Lassen Sie uns lieber nach anderen Möglichkeiten suchen, dem depressiven Patienten zu helfen, die Schwelle zum Besuch von Fachpraxen und Institutsambulanzen zu senken und Berührungsängste abzubauen. Die Schulung der Hausärzte in der Diagnostik von depressiven Zuständen, deren Bewertung und der Beurteilung des jeweiligen Schweregrades wäre der Behandlung dieser Patienten dienlicher als die Anwendung eines telefonischen Assessments.

\section{Literatur}

1 Loeb P. Unbefriedigende Behandlung der Depression - nur Sache des Hausarztes? Schweizerische Ärztezeitung 2010; 91: 4

2 Gensichen J, Huchzermeier C, Aldenhoff JB et al. Signalsituationen für den Beginn einer strukturierten Depressionsdiagnostik in der Allgemeinarztpraxis - Eine praxis-kritische Einschätzung internationaler Leitlinien. Z Arztl Fortbild Qualitatssich 2005; 99: 57-63

3 Torge M, Peitz M, Beyer $M$ et al. Am Anfang stehen oft nur diskrete Signale - Depressiv Erkrankte erkennen und behandeln. Der Hausarzt 2006; 5: 40-44

4 Tyrer $P$. Are general practitioners really unable to diagnose depression? Lancet 2009; 347: 589-590

5 DGPPN, BÄK, KBV, AWMF, AkdÄ, BPtK, BApK, DAGSHG, DEGAM, DGPM, DGPS, DGRW (Hrsg) für die Leitliniengruppe Unipolare Depression. S3Leitlinie/Nationale VersorgungsLeitlinie Unipolare Depression-Langfassung, 1. Auflage 2009. Berlin, Düsseldorf: DGPPN, ÄZQ AWMF, 2009 Available from: http://www.dgppn.de, http://www.versorgungs leitlinien.de, www.awmf-leitlinien.de

6 Klesse C, Berger M, Bermejo I et al. Evidenzbasierte Psychotherapie der Depression - Therapiepraxis nach der aktuellen S3-/Nationalen Versorgungsleitlinie „Unipolare Depression“. Psychotherapeut 2010; 55: 247-263

7 Gensichen J, Korff M von, Muth C et al. Case management for depression by health care assistants in small primary care practices - a cluster randomized trial. Ann Intern Med 2009; 151: 369-380

8 Schulberg HC, Bruce ML, Lee PW et al. Preventing suicide in primary care patients: the primary care physician's role. Gen Hosp Psychiatry 2004; 26: 337-345

9 Deutsches Bündnis gegen Depression e.V. Available from: http://www. buendnis-depression.de 